\title{
Optimized Multichannel Filter Bank with Flat Frequency Response for Texture Segmentation
}

\author{
Nezamoddin N. Kachouie \\ Department of Systems Design Engineering, University of Waterloo, 200 University Avenue West, \\ Waterloo, ON, Canada N2L $3 G 1$ \\ Email: nnezamod@engmail.uwaterloo.ca \\ Javad Alirezaie \\ Department of Systems Design Engineering, University of Waterloo, 200 University Avenue West, \\ Waterloo, ON, Canada N2L $3 G 1$ \\ Email: javad@rousseau.uwaterloo.ca \\ Electrical and Computer Engineering Department, Ryerson University, 350 Victoria Street, \\ Toronto, ON, Canada M5B 2 K3 \\ Email: jalireza@ee.ryerson.ca
}

Received 17 May 2004; Revised 10 March 2005; Recommended for Publication by Marc Moonen

\begin{abstract}
Previous approaches to texture analysis and segmentation use multichannel filtering by applying a set of filters in the frequency domain or a set of masks in the spatial domain. This paper presents two new texture segmentation algorithms based on multichannel filtering in conjunction with neural networks for feature extraction and segmentation. The features extracted by Gabor filters have been applied for image segmentation and analysis. Suitable choices of filter parameters and filter bank coverage in the frequency domain to optimize the filters are discussed. Here we introduce two methods to optimize Gabor filter bank. First, a Gabor filter bank with a flat response is implemented and the optimal feature dimension is extracted by competitive networks. Second, a subset of Gabor filter bank is selected to compose the best discriminative filters, so that each filter in this small set can discriminate a pair of textures in a given image. In both approaches, multilayer perceptrons are employed to segment the extracted features. The comparisons of segmentation results generated using the proposed methods and previous research using Gabor, discrete cosine transform (DCT), and Laws filters are presented. Finally, the segmentation results generated by applying the optimized filter banks to textured images are presented and discussed.
\end{abstract}

Keywords and phrases: filter bank, Gabor, DCT, multilayer perceptron, competitive network, texture segmentation.

\section{INTRODUCTION}

Texture segmentation and analysis is an important aspect of pattern recognition and digital image processing. In image analysis, textures have been used to perform scene segmentation for object and region recognition, surface classification, and shape recognition. Texture segmentation involves accurately partitioning an image into sections according to the textured regions or recognizing the borders between different textures in the scene or image.

Several researchers in this field $[1,2,3,4,5,6]$ have proposed texture segmentation and analysis methods using a filter bank model which is based on the human vision system's (HVS) unique capabilities for texture segmentation $[7,8]$. In this model, a set of filters in the frequency domain (or a set of masks in the spatial domain) are applied in parallel to an input image which decomposes it into a set of filtered images. The set of filtered images are used directly as feature images, or they are processed and/or combined to extract the features. Eventually extracted features are used for the segmentation of the input image. Each individual filter in the bank is designed to focus on a specific local spatial region and a specific range of frequency to make spatial/spatial-frequency decomposition possible. The joint spatial/spatial-frequency decomposition, unlike Fourier analysis, considers the local frequency variations that vary with position in the image. Textured images that are encoded in narrow spatial frequencies and orientation channels can be recognized and segmented by filtered images that carry out the texture features. These features describe local spatial-frequencies and orientations to which the channels are tuned. Therefore, texture can be regarded as carrying region information and the local structure of a texture can be characterized by the frequencies and orientations of the carrier that is contained in the channel outputs. 
There are several suitable ways to implement filter banks which can be used for textured images. From a practical point of view, some filters may be more useful for specific texture segmentation tasks but may not be suitable for others. Exponentially and/or sinusoidally modulated Gaussian filters, also known as Gabor filters, have proven to be very useful for texture analysis $[1,2,9,10]$. While other filter banks can perform joint spatial/spatial-frequency decomposition, a filter bank using a Gabor base function is one of the most attractive. This set of filters has an optimal localization in the joint spatial/spatial-frequency domain according to the uncertainty principle $[1,2]$. These selective bandpass filters with different radial spatial frequencies and orientations have an optimum resolution in the time and frequency domains that resembles the simple visual cortical cell characteristics $[7,8]$.

This paper describes two texture segmentation approaches using the general multichannel decomposition approach and employing neural networks both for feature reduction and classification. By applying a Gabor filter bank to the input image with suggested radial frequencies and orientations, a set of filtered images is generated. The multichannel decomposition is accomplished by estimating the local energy in the filter outputs. Having five radial frequencies and eight orientations generates forty feature images. To reduce the features and obtain an optimal feature dimension, we trained a competitive network $(\mathrm{CN})$ with an unsupervised learning method. The weight vectors of the trained network are used to reduce the feature dimension. Eventually the resultant reduced features are used to train a multilayer perceptron with a supervised learning method to segment the input image.

This paper is organized as follows. In Section 2, previous works using Gabor filter banks and the multichannel decomposition by Gabor filters are presented. Section 3 gives a detailed description of the first proposed method using Gabor filter bank in conjunction with a competitive network (GCN).

In Section 4, the second proposed method using a subset of Gabor filter bank is explained. In Sections 5 and 6, both the results and conclusion are presented.

\section{GABOR FILTER BANK AND MULTICHANNEL DECOMPOSITION}

Considerable research has been performed using Gabor filters for texture segmentation and analysis $[1,3,4,9,11]$. A brief overview of the previous work is presented in this section as following.

\subsection{Previous works using Gabor filter bank}

The research involving the use of Gabor filters for texture segmentation can be divided into three major disciplines:

(1) investigating the best frequencies and orientations (bandwidth) according to the characteristics of a specific textured image;

(2) inventing robust feature extraction and feature reduction methods;
(3) employing the best classification and segmentation methods to devote to the optimal extracted features.

Bovik et al. [1] have proposed a computational approach for analyzing visible textures. In their method, boundaries are detected between textures by comparing the channel amplitude responses and detecting discontinuities in the texture phase by locating large variations in the channel phase responses. Dunn and Higgins have investigated the optimal Gabor filters [2] and argued that Gabor filter outputs can be presented as a Rician model. They have developed an algorithm to select optimal filter parameters to discriminate texture pairs. Jain and Farrokhnia proposed a filter selection method [3] based on reconstruction of the input image from the filtered images. They also proposed the optimum radial frequencies and orientations for different channels. These have been used widely by many researchers. Unser and Eden described an unsupervised texture segmentation method [9] using the Karhunen-Loeve transform on the resulting features of Gabor filters to reduce the feature vector dimension. Weldon et al. presented a method [10] to design a single Gabor filter for multitextured image segmentation. Clausi and Jernigan investigated and compared different techniques [11] used to extract texture features and Jain and Karu proposed a neural network texture classification method [12] as a generalization of the multichannel filtering method. Despite all the research on optimizing Gabor filters for texture segmentation, this area is still developing rapidly. Since there are several unknowns in Gabor filter implementation, a more accurate selection from a wide range of values for each parameter and combination of these values have a crucial importance in designing a filter bank for a specific texture segmentation problem. On the other hand, despite its very attractive features, a Gabor filter bank does not provide a flat frequency response, and some frequency bands are not covered adequately. Hence, implementing a Gabor filter bank with a flat response not only would utilize the attractive features of a Gabor filter bank, but also would provide better segmentation results by including the usually missed frequency bands. The proposed algorithm is implemented to optimize Gabor filter bank for texture segmentation and is successfully applied to synthetic texture images.

\subsection{Multichannel decomposition by Gabor filter bank}

A typical Gabor filter in the spatial and the spatial-frequency domain is depicted in Figure 1. A Gabor base function is a Gaussian function modulated with an exponential or sinusoidal function that is defined in terms of the product of a Gaussian and an exponential. Two-dimensional Gabor functions $h(x, y)$ can be written as

$$
h(x, y, \theta)=g(x, y) \exp \left(2 \pi j f_{0} x_{\theta}\right),
$$

where

$$
g(x, y)=\frac{1}{2 \pi \sigma_{x} \sigma_{y}} \cdot \exp \left\{-\frac{1}{2}\left(\frac{x^{2}}{\sigma_{x}^{2}}+\frac{y^{2}}{\sigma_{y}^{2}}\right)\right\},
$$




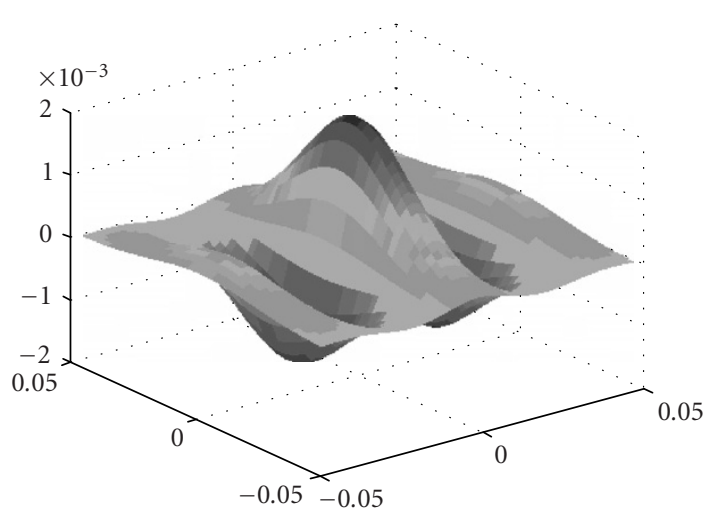

(a)

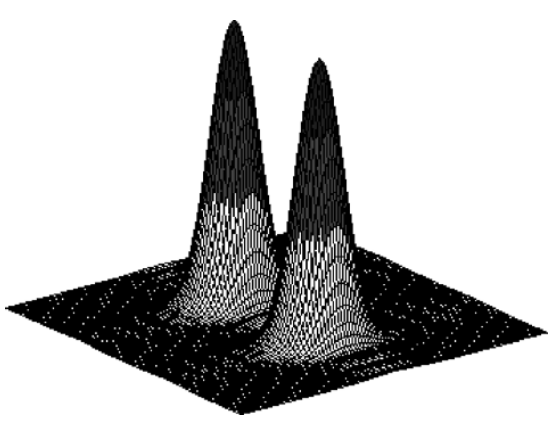

(b)

FIGURE 1: A typical Gabor filter in (a) spatial domain and (b) spatial-frequency domain.

and its frequency response $H(u, v)$ is

$H(u, v)=G\left(u-f_{0}, v\right)=\exp \left\{-2 \pi^{2}\left[\left(u-f_{0}\right)^{2} \sigma_{x}^{2}+v^{2} \sigma_{y}^{2}\right]\right\}$,

where $f_{0}^{2}=u_{0}^{2}+v_{0}^{2}, \theta=\tan ^{-1}\left(v_{0} / u_{0}\right), x_{\theta}=x \cos \theta+y \sin \theta$, and $y_{\theta}=-x \sin \theta+y \cos \theta$.

Gabor functions are bandpass filters which are Gaussian, centered on $\left(f_{0}, \theta\right)$ in the spatial-frequency domain. The parameters $f_{0}, \theta, \sigma_{x}$, and $\sigma_{y}$ determine the subband Gabor filter; $f_{0}$ and $\theta$ are center frequency and orientation and $\sigma_{x}$ and $\sigma_{y}$ are the bandwidths of the filter in the $x$ and $y$ directions [13]. Equation (1) defines a complete Gabor function consisting of both real and imaginary (or even and odd) components. Rotation by $\theta$ in the spatial domain ( $x-y$ plane) or in the spatial-frequency domain ( $u-v$ plane) provides selective arbitrary orientation for different channels. We can implement a Gabor filter bank by using only even-symmetric or real components as suggested by Jain and Farrokhnia [3] and can be represented by

$$
h(x, y)=g(x, y) \cdot \cos \left\{2 \pi f_{0} x\right\}
$$

and $H(u, v)$ is

$$
H(u, v)=G\left(u-f_{0}, v\right)+G\left(u+f_{0}, v\right),
$$

and is composed of two Gaussians in the spatial-frequency domain compared with one Gaussian in the complex version.

General multichannel filtering methods as shown in Figure 2 consist of three major stages: usage of the filter bank to generate filtered images, local energy estimation for feature extraction, and classification of extracted features into different regions for segmentation [14]. In the first step, a textured input image is decomposed into filtered images. Typically, in the second stage, a local energy function consisting of nonlinearity and smoothing is applied on the filtered images (the output of the Gabor filter bank) for feature extraction $[1,3,10,11]$. The most well-known nonlinearity

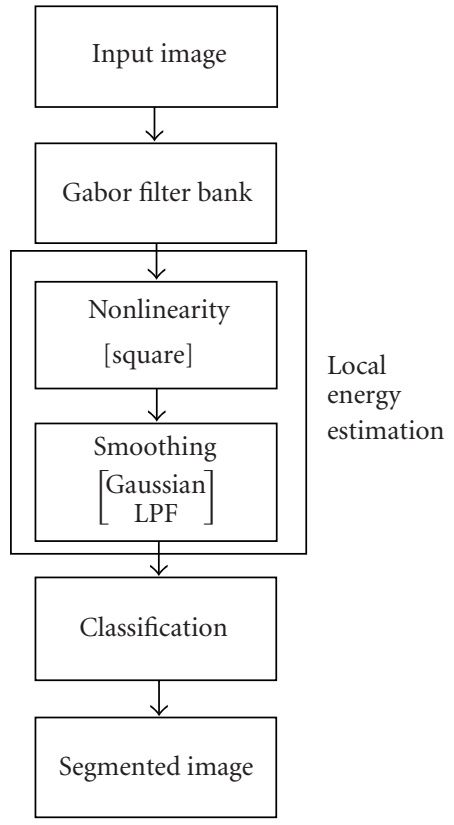

Figure 2: Multichannel decomposition process.

functions are (1) sigmoid function, (2) rectifying, (3) square function, (4) magnitude response, and (5) real part. In this step, each channel corresponding to a different filter is tuned to a different radial frequency or orientation to capture local characteristics of different textures in the input image such as spatial frequency, edge intensity, or direction [15]. After the second stage, a set of feature images or a feature vector corresponding to each pixel in the input image is generated. The dimension of feature vectors is equal to (or multiplied by an integer) the number of filters in the filter bank. Eventually the feature vectors should be classified and assigned to different textures. There are several classification methods [16] to accomplish the segmentation task such as Bayesian classifier, nearest neighbor classifier, multilayer perceptron (MLP), Fisher linear discriminant (FLD), and learning vector quantization (LVQ). 


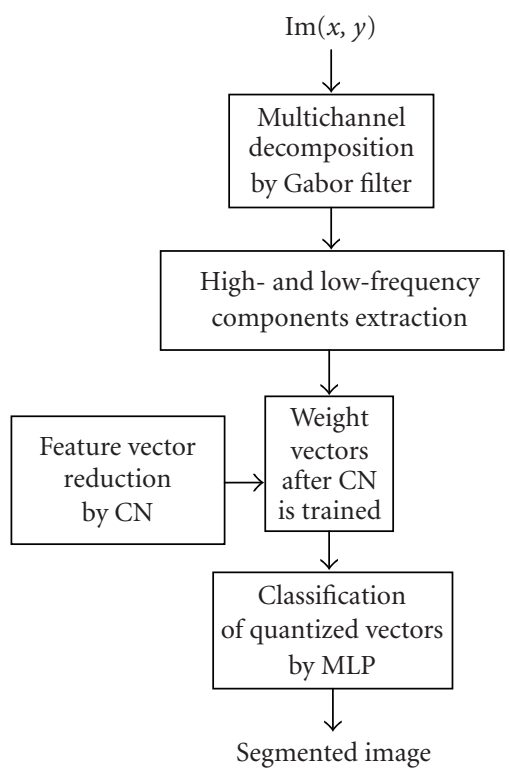

FIGURE 3: GCN method.

\section{GABOR FILTER BANK IN CONJUNCTION WITH COMPETITIVE NETWORK (GCN)}

The first proposed method (GCN) as shown in Figure 3 consists of the following stages: multichannel decomposition, high- and low-frequency component extraction, computing features, feature vector reduction, and classification by multilayer perceptron. The detail of each stage is described in this section.

\subsection{Multichannel decomposition}

This stage consists of filtering by a Gabor filter bank, applying a nonlinearity function, and smoothing the results. We used three sets of Gabor filters composed of 20, 30, and 40 filters. For all sets, the same five radial frequencies suggested by Jain and Farrokhnia [3], that is, $4 \sqrt{2}, 8 \sqrt{2}, 16 \sqrt{2}, 32 \sqrt{2}, 64 \sqrt{2}$, are used.

The radial frequency bandwidth is one octave, thus the frequency difference of $f_{1}$ and $f_{2}$ is given by $\log _{2}\left(f_{2} / f_{1}\right)$, and is equal to 1 . In the first bank for each radial frequency, four orientations $0^{\circ}, 45^{\circ}, 90^{\circ}$, and $135^{\circ}$ are used to generate the total number of 20 channels. In the second bank, six orientations $0^{\circ}, 30^{\circ}, 60^{\circ}, 90^{\circ}, 120^{\circ}$, and $150^{\circ}$ are used that give a bank of 30 filters, and the third one composed of 40 filters is obtained by eight orientations for each radial frequency, that is, $0^{\circ}, 22.5^{\circ}, 45^{\circ}, 67.5^{\circ}, 90^{\circ}, 112.5^{\circ}, 135^{\circ}$, and $157.5^{\circ}$.

The filter banks consisting of 20 and 30 filters using $45^{\circ}$ and $30^{\circ}$ orientations, respectively, were used widely in previous research $[3,4,11,12]$. Here we introduce the third filter bank using $22.5^{\circ}$ angular bandwidths and orientations. In several experiments with a variety of different textures using all three filter banks, we computed the preserved energy in the filtered images. By comparing and sorting the filtered images according to the preserved energies, we observed that by dividing the angular bandwidth by two, that is, $22.5^{\circ}$ in place of $45^{\circ}$ and covering the frequency domain by this angu- lar bandwidth, not only could we catch the textures with $45^{\circ}$ orientations, that is, $0^{\circ}, 45^{\circ}, 90^{\circ}$, and $135^{\circ}$, but also we could better discriminate and classify textures with $22.5^{\circ}$ orientations, that is, $0^{\circ}, 22.5^{\circ}, 67.5^{\circ}, 112.5^{\circ}$, and $157.5^{\circ}$. It was noticed that for some textures, maximum energy is preserved by filters with $45^{\circ}$ orientations (and $22.5^{\circ}$ angular bandwidths) and filters with $22.5^{\circ}$ orientations (and $22.5^{\circ}$ angular bandwidths). By applying $22.5^{\circ}$ orientations for different radial frequencies and sorting the filtered images regarding preserved energies, we observed that some of the filtered images with these orientations are among the ten best filters with respect to the preserved energy of the original image. Hence, for this filter bank, we used eight equally spaced orientations $0^{\circ}, 22.5^{\circ}, 45^{\circ}, 67.5^{\circ}, 90^{\circ}, 112.5^{\circ}, 135^{\circ}$, and $157.5^{\circ}$ and to ensure proper coverage of the spatial-frequency domain, we set the angular bandwidth to $22.5^{\circ}$. Orientations and radial frequencies of the ten best filters using the three mentioned filter banks are presented in Table 1. The best filters are selected and sorted according to the preserved energies. The results are obtained by applying three Gabor filter banks to a complex synthetic textured image depicted in Figure 4.

In the second step, a square function is applied to achieve nonlinearity and it causes the sinusoidal modulations in the output of a filter bank to be transformed to a square modulation. To smooth out the fluctuations in the specific texture or noise in the image " $\operatorname{Im}(x, y)$," a Gaussian lowpass filter is applied to the output of the filter bank. The size of the smoothing function is determined according to the size of the Gabor bandpass filter. The impulse response of the Gaussian filter is

$$
h(x)=\frac{1}{\sqrt{2 \pi} \sigma} \cdot \exp \left\{-\frac{1}{2}\left(\frac{x^{2}}{\sigma^{2}}\right)\right\},
$$

where $\sigma=1 / 2 \sqrt{2} f_{0}$ and $f_{0}$ is the radial frequency of the bandpass Gabor filter.

\subsection{High- and low-frequency component extraction}

As depicted in Figure 5, neither this filter bank having 40 Gabor filters nor the two other Gabor filter banks with 20 and 30 filters completely cover the corners of the frequency domain along the diagonals. The coverage of the Gabor filter banks are not perfectly flat, thus there are some regions that are not covered and as a result do not have a flat response, including very high-frequency and very low-frequency regions. In the proposed method, the spatial implementation of the Gabor filter bank is optimized by considering the diagonal high-frequency and very low-frequency components.

The low frequency components that are not included in the Gabor filter bank are obtained by (4) where radial frequency $f_{0}$ is equal to zero and concludes a Gaussian lowpass filter as follows:

$$
h_{2}(x, y)=g(x, y) \cdot \cos \left\{2 \pi f_{0} x\right\}=g(x, y) .
$$

To obtain the optimized filter bank as depicted in Figure 6, the high-frequency components are computed by using the Gabor filter bank and Gaussian lowpass filter. 
TABLE 1: Radial frequencies and orientations of the 10 best filters of the 3 filter banks.

\begin{tabular}{ll}
\hline Filter bank & Radial frequency and orientation of 10 best filters \\
\hline 1: angular band width $=45^{\circ}$ & $64 \sqrt{2} \& 90^{\circ}, 64 \sqrt{2} \& 135^{\circ}, 64 \sqrt{2} \& 0^{\circ}, 64 \sqrt{2} \& 45^{\circ}, 32 \sqrt{2} \& 90^{\circ}$, \\
& $32 \sqrt{2} \& 0^{\circ}, 32 \sqrt{2} \& 135^{\circ}, 32 \sqrt{2} \& 45^{\circ}, 16 \sqrt{2} \& 0^{\circ}, 16 \sqrt{2} \& 45^{\circ}$ \\
\hline 2: angular band width $=30^{\circ}$ & $64 \sqrt{2} \& 90^{\circ}, 64 \sqrt{2} \& 150^{\circ}, 64 \sqrt{2} \& 0^{\circ}, 64 \sqrt{2} \& 120^{\circ}, 64 \sqrt{2} \& 60^{\circ}$, \\
& $32 \sqrt{2} \& 90^{\circ}, 64 \sqrt{2} \& 30^{\circ}, 32 \sqrt{2} \& 0^{\circ}, 32 \sqrt{2} \& 60^{\circ}, 32 \sqrt{2} \& 150^{\circ}$ \\
\hline 3: angular band width $=22.5^{\circ}$ & $64 \sqrt{2} \& 90^{\circ}, 64 \sqrt{2} \& 157.5^{\circ}, 64 \sqrt{2} \& 135^{\circ}, 64 \sqrt{2} \& 67.5^{\circ}, 64 \sqrt{2} \& 112.5^{\circ}$, \\
& $64 \sqrt{2} \& 0^{\circ}, 32 \sqrt{2} \& 90^{\circ}, 64 \sqrt{2} \& 45^{\circ}, 64 \sqrt{2} \& 22.5^{\circ}, 32 \sqrt{2} \& 67.5^{\circ}$ \\
\hline
\end{tabular}

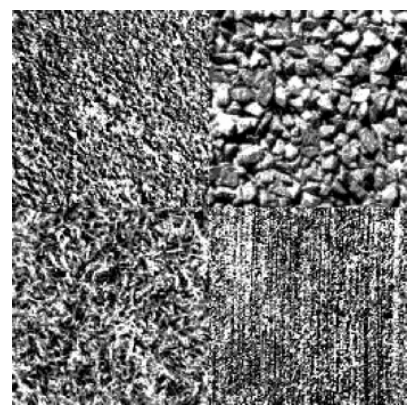

Figure 4: Textured image composed of Asphalt.0000, Misc.0002, Grass.0002, and Concrete.0001 selected from MeasTex Image Texture Database.

Each high-frequency component is computed by

$$
\begin{aligned}
h_{d}(x, y)= & \operatorname{Im}(x, y) * \delta(x, y)-h_{2}(x, y) * \operatorname{Im}(x, y) \\
& -\sum_{1}^{L} h_{1}(x, y) * \operatorname{Im}(x, y),
\end{aligned}
$$

where $h_{d}(x, y)$ is the diagonal high-frequency component and is equal to $h(x, y) * \operatorname{Im}(x, y), h_{1}$ is the Gabor filter impulse response, $L$ is the Number of filters in the filter bank, and $h_{2}$ is the Gaussian lowpass impulse response.

\subsection{Computing features}

To compute the feature vector for each pixel of the input image, the mean and variance are computed over a neighborhood window of the pixel [17]. Mean and variance are reliable attributes of textures and have small variations over the same texture when the window size is accurately selected to cover the texture periodicity. According to the periodicity of the textures in the input image, the size of the neighborhood window could change and be determined by the user. For textured images with smaller periodicity, the window centered by the regarded pixel would be smaller and would select the larger window for the textures with larger periodicity. In the experiments, window sizes from as small as 4 by 4 to as big as 32 by 32 pixels are used depending on texture periodicity.

\subsection{Feature vector reduction by competitive networks}

Grouping several neurons in a single layer [18, 19] forms a competitive network. Each neuron is a single processing element that shares its processing functions with other neurons

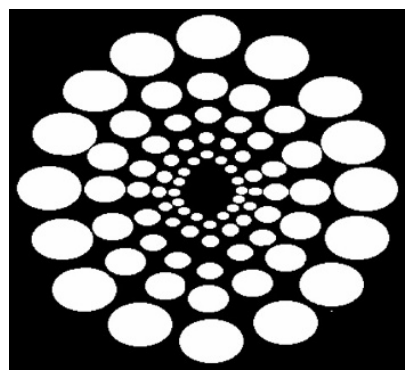

Figure 5: A bank of 40 Gabor filters.

and responds to a group of input vectors in a different cluster maximally. This layer of neurons can classify any input vector, since the neuron with the strongest response for a given input identifies the cluster to which the input vector is most likely to belong. Since, for this layer there is no external judge to decide which neuron wins, the neurons must decide this by themselves $[18,19]$ by using an unsupervised learning method. This decision-making process needs communication among all neurons in the layer.

To reduce the feature vector dimension a competitive network is used. In the competitive layer the neurons are distributed in order to recognize frequently presented input vectors in an unsupervised manner. The competitive transfer function accepts a net input for each neuron in the layer and returns neuron outputs of 0 for all neurons except for the winner, which outputs 1 . In a competitive layer each neuron competes to respond to an input vector $p$; the neuron whose weight vector is closest to $p$ gets the highest net input and, therefore, wins the competition and outputs 1 and all other neurons output 0 .

In the training phase, the winner is moved closer to the input by adjusting the weights of the winning neuron [18] with the learning rule as shown by

$$
\Delta w=\lambda(p-w)
$$

where $p, w$, and $\lambda$ are an input vector, an input weight vector, and a learning rate, respectively. Since this learning rule allows the weights to learn the input vector, it is useful in recognition applications. Thus, the neuron whose weight vector is the closest to the input vector will be updated to be even closer. As a result the winning neuron is more likely to win the competition when a similar vector is provided, and less 


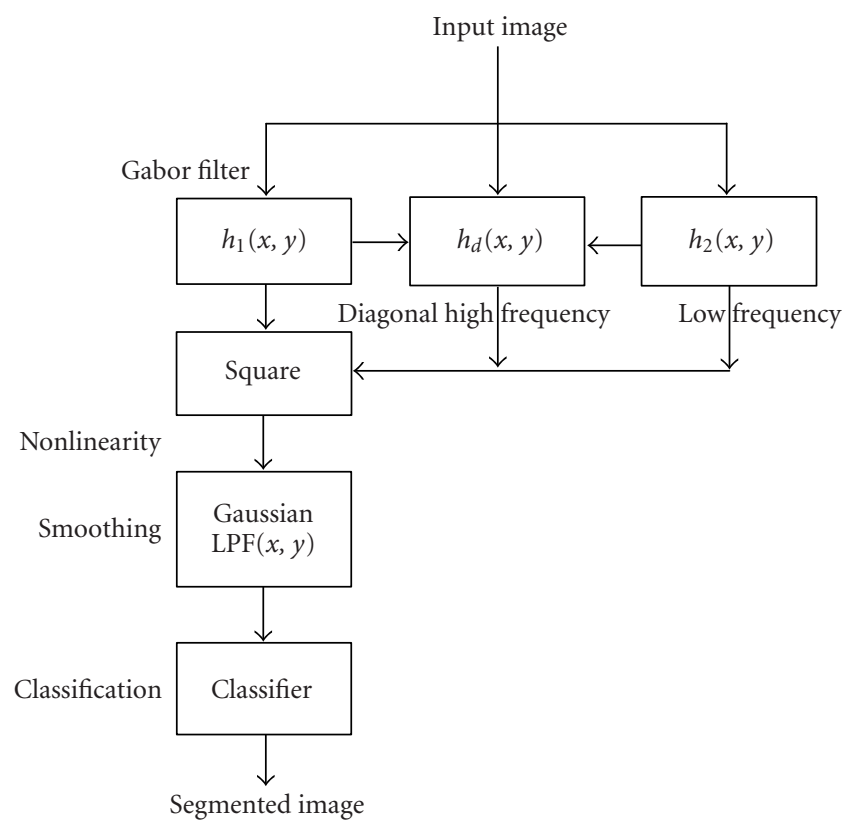

Figure 6: Optimized filter bank.

likely to win if a very different input vector is provided. During training, as more inputs are presented, each neuron in the layer closest to a group of input vectors adjusts its weights to more closely resemble the inputs. Eventually, every cluster of similar input vectors will have a neuron that identifies the presented vector if it belongs to the cluster and the competitive network will categorize the input vectors.

To reduce the feature dimension in the GCN method, a competitive network $(\mathrm{CN})$ as depicted in Figure 7 is used. The motivation to use competitive networks stems from the previous research using LVQ for classification [4, 18]. LVQ consists of one competitive layer and one linear layer, and it is a supervised learning algorithm. LVQ describes the class borders by the nearest neighbor rule and its main applications are in statistical pattern recognition and classification [18]. Despite using a hidden competitive layer, LVQ is restricted by only learning linear relationships between quantized vectors and desired output vectors. If the network could learn the nonlinear relationships between the reduced dimension vectors and desired outputs, improved segmentation results would possibly be obtained .

On the other hand, applying the resultant weight vectors of a competitive layer to the features prior to feeding the classifier would reduce the dimensionality of feature vectors as depicted in Figure 7 and faster segmentation would result. In order to train $\mathrm{CN}$, sample vectors are selected at random among the extracted feature vectors obtained by the Gabor filter bank in the previous step. After $\mathrm{CN}$ is trained, the weight vectors of the layer are regarded as quantizing mask coefficients. These coefficients are the optimum extracted features of the Gabor filter bank. To select the number of neurons in the network that is equal to the number of the most important eigenvectors, 42 neurons (the total num-

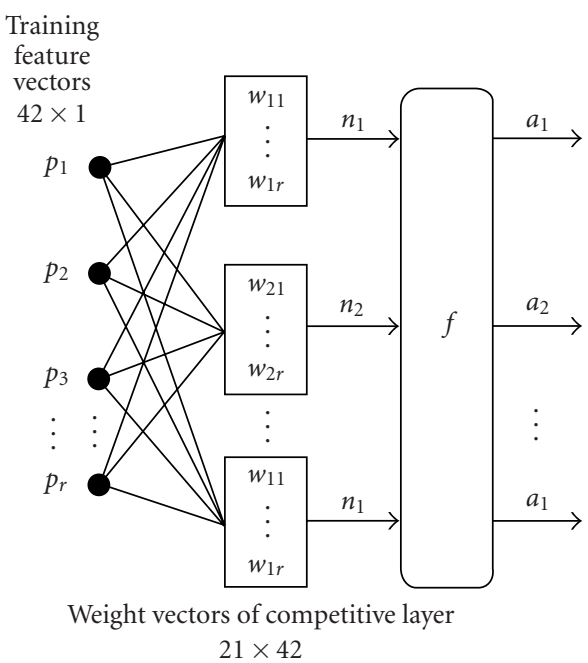

(a)

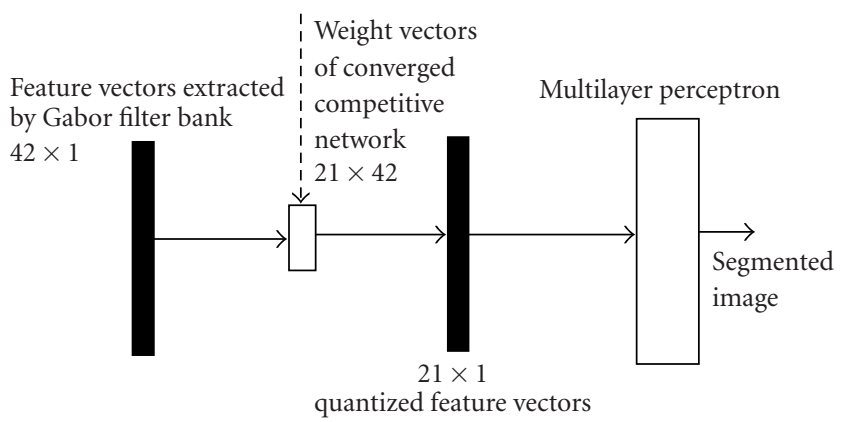

(b)

FIGURE 7: Feature reduction and classification by GCN method.

ber of possible eigenvectors) are selected as the initial value, then the layer is trained with training samples and the classification error for the network is computed. By pruning the neurons in the network, repeating the same approach, and computing the classification error, the appropriate number of neurons in the layer is chosen. In our competitive network according to the classification error and dimension of quantized feature vectors, 21 neurons are selected for $\mathrm{CN}$ as the optimum number of neurons. After selecting the appropriate number of neurons, the competitive layer will be trained and the estimated eigenvectors (i.e., weight vectors of the network) are obtained and regarded as quantizing mask coefficients. These coefficients are applied on the extracted feature vectors by the Gabor filter bank. The resultant weight vectors of a competitive layer are applied to the features prior to feeding in the classifier and will reduce the dimensionality of feature vectors.

\subsection{Classification by multilayer perceptron}

A multilayer perceptron (MLP) has multiple layers with nonlinear transfer functions to learn the nonlinear relationships between input and output vectors. Feedforward networks 
often have one or more hidden layers of sigmoid neurons followed by an output layer [19]. The MLP is trained by adjusting the weights using least-square error (LSE) that minimizes the mean square error as shown by

$$
E=\frac{\sum_{1}^{N}\left(R_{k}-O_{k}\right)^{2}}{N}
$$

The total square error between the desired $R_{k}$ class and the actual output $O_{k}$ is calculated for $N$ neurons in output layer $K$. To train the neural network, the gradient is determined by using a backpropagation technique which involves performing computations backwards through the network. After the backpropagation network is trained properly, it typically provides reasonable answers when presented with inputs that it has never seen. Commonly, a new input vector similar to past inputs used during training leads to an output similar to correct values. A 3-layer perceptron, which is used to accomplish the segmentation task, is depicted in Figure 7. Our MLP uses the sigmoid transfer function in all three layers. During training, randomly selected quantized feature vectors are assigned to proper classes. Although there are 42 filters in the filter bank, the quantized feature dimension is 21. After MLP is trained, input images will be segmented by assigning quantized feature vectors to proper regions.

CN and MLP are used in the proposed method for feature dimension reduction and classification, respectively; therefore we need a two-stage training process. In the first stage, an unsupervised learning using unlabeled samples is employed to train the competitive network. As an efficient approach to compute principle component analysis (PCA), the weight vectors of the trained network are applied to the extracted Gabor features before classification. In the second stage, a supervised learning is employed to train the MLP, that is, classifier, by labeled samples. Although in this stage the same set of training samples as of the first stage are used, the label of each sample is also required to train MLP. Using labeled samples, MLP adjusts its weight vectors such that it can effectively recognize all different classes (labels). Despite having two independent training stages, the classification performance of MLP depends on how precise CN approximates PCA which in turn depends on training samples and initialization of the weight vectors.

\section{ADAPTIVELY SELECTED GABOR FILTER BANK}

The number of filters in a filter bank affects classification performance and has a crucial importance. Much research has been conducted to reduce the number of required filters in the Gabor filter bank for segmentation. In this section, an algorithm is presented to select an optimized subset of the Gabor filter bank. The proposed method as depicted in Figure 8 consists of three stages: (1) image decomposition, (2) subset filter selection, and (3) classification. The decomposition and classification stages are the same as the first approach; hence the second stage is explained in the following section.

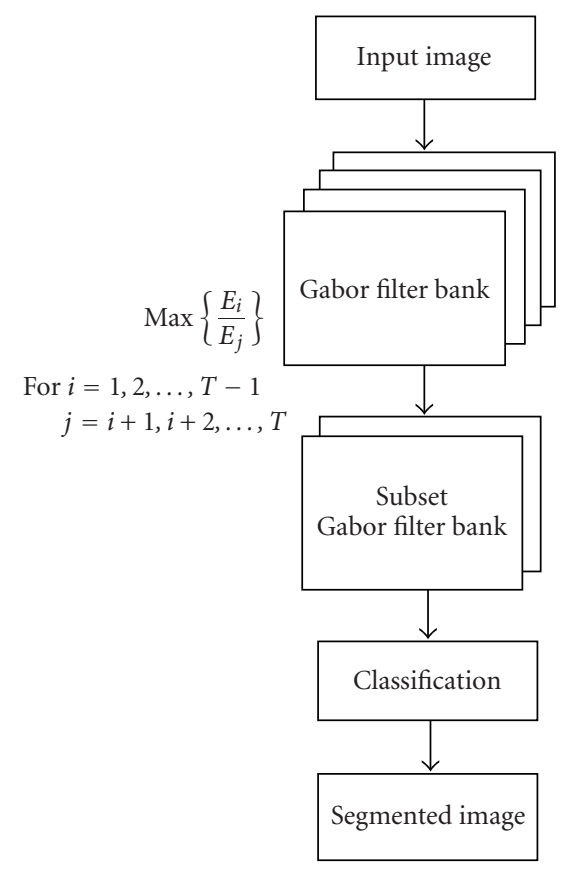

FIgURE 8: Adaptive filter selection.

\subsection{Selection of a subset bank of the Gabor filters}

In this approach, a set of Gabor filters are selected as the best Gabor filters, so that each single Gabor filter in this small set of selected Gabor filters can discriminate a pair of textures in an image with multiple textures. Assume that an input image $I$ consists of $T$ different textural classes $T_{c}, c=1,2, \ldots, T$, and let $I_{c}$, a subimage of $I$, be a training sample of the texture class $T_{c}$. Furthermore, assume that $E_{i}$ is the preserved energy of the texture class $i$. The optimal Gabor filter set is selected based on the energy ratio of each distinct texture pair $T_{i}$ and $T_{j}, i \neq j$ :

$$
\frac{E_{i}}{E_{j}}, \quad i=1,2, \ldots, T-1, j=i+1, i+2, \ldots, T .
$$

Therefore, the optimal filter set is given by

$\operatorname{Maximum}\left\{\frac{E_{i}}{E_{j}}\right\}, \quad i=1,2, \ldots, T-1, j=i+1, i+2, \ldots, T$.

In this approach, the best Gabor filters are selected so that the corresponding energy ratio is maximum for each pair of distinct textures in a multiple-textured image. For instance, having an image composed of two different texture classes $T_{i}=1$ and $T_{j}=2$, the filter set includes only one filter to discriminate these two textures and has the maximum energy ratio as Maximum $\left\{E_{1} / E_{2}\right\}$.

To choose the best filter by obtaining the maximum energy ratio, the energy ratio $E_{i} / E_{j}$ is calculated for all 40 Gabor filters with $22.5^{\circ}$ angular bandwidth and the following radial 
frequencies:

$$
4 \sqrt{2}, 8 \sqrt{2}, 16 \sqrt{2}, 32 \sqrt{2}, 64 \sqrt{2} .
$$

The 40 obtained energy ratios are sorted accordingly and the filter which has the maximum ratio among them is selected for each pair of textures:

$$
\begin{gathered}
\left\{\frac{E_{1}}{E_{2}}\right\}_{f}, f=1,2, \ldots, 40, \\
\left\{\frac{E_{1}}{E_{2}}\right\}_{i}>\left\{\frac{E_{1}}{E_{2}}\right\}_{j}>\cdots>\left\{\frac{E_{1}}{E_{2}}\right\}_{k},
\end{gathered}
$$

where, $i, j, k=1,2, \ldots, 40$ and $i \neq j, i \neq k, j \neq k$.

The energy ratio $E_{i} / E_{j}$ of each texture pair $T_{i}$ and $T_{j}$, $i \neq j$, for an image containing $T$ different textures is calculated on each filtered image and sorted in decreasing order so that the maximum ratio is achieved according to (12). The larger the value of ratio $E_{i} / E_{j}$, the better the discrimination of distinct texture pair $i$ and $j$. Thus the best discriminative filter for each pair of different textures according to the maximum energy ratio is selected to be included in the subset filter bank:

$$
\begin{aligned}
& f_{\text {index }} \in \operatorname{SFB}\{\text { Subset Filter Bank }\}, \\
& \text { if }\left\{\frac{E_{i}}{E_{j}}\right\}_{\text {index }}>\left\{\frac{E_{i}}{E_{j}}\right\}_{k} \quad \forall k \in 1,2, \ldots, 40, \text { index } \neq k, \\
& \text { for } i=1,2, \ldots, T-1 ; j=i+1, i+2, \ldots, T .
\end{aligned}
$$

The subset filter is composed of $N$ filters:

$$
N=\frac{T \times(T-1)}{2} .
$$

For instance the size of subset filter for an image containing 4 different textures is

$$
T=4 \Longrightarrow N=\frac{4 \times(4-1)}{2}=6 .
$$

\section{RESULTS}

In several experiments, three sets of filters are used consisting of 20,30 , and 40 filters with $45^{\circ}, 30^{\circ}$, and $22.5^{\circ}$ orientations, respectively. Applying the first method, the segmented results are obtained using 40 filters with $22.5^{\circ}$ angular bandwidths. Two new low-frequency and high-frequency filters are added to improve the filter bank. This raises the total number of filters in the filter bank to 42 . The increase in the feature dimension by using 42 filters in comparison with 20 and 30 filters in the previous published reports is compensated by feature quantization using competitive network. The dimension of quantized feature vectors is 21 and it may change depending on the number of textures in the image, that is, if we have less than 4 textures in the input image, we could use a smaller dimension for quantized vectors. In the second approach to segment the textured images, a subset filter bank is adaptively selected. The Gabor filters which compose this subset filter are selected so that in a multiple texture image, the energy ratio of any two distinct textures is maximum. The number of filters in this subset is variable and depends on the number of textures in the image.

In this section, some results obtained by applying the proposed approaches and three widely used approaches for texture segmentation including Gabor, discrete cosine transform (DCT), and Laws filters are presented. Since the Gabor filter was outperformed in comparison with the Laws and DCT in previous research [4], optimized Gabor filter banks will be compared with these filter banks to evaluate the performance of the proposed methods. As it was explained in Section 4.1, Ng et al. [5] introduced a 3 by 3 DCT to extract features from textured images. They suggested using eight masks excluding the low-frequency component of the DCT. On the other hand, Laws introduced a separable filter bank to identify different textures for texture segmentation. The suggested filter bank by Laws [6] is composed of 25 filters including five filters in each dimension. These onedimensional kernels are

$$
\begin{gathered}
L 5=[1,4,6,4,1], \quad E 5=[-1,-2,0,2,1], \\
S 5=[-1,0,2,0,-1], \quad W 5=[-1,2,0,-2,1], \\
R 5=[1,-4,6,-4,1] .
\end{gathered}
$$

By applying the filter bank to the input image, 25 filtered images are produced. These mnemonics $(L, E, S, W$, and $R)$ stand for level, edge, spot, wave, and ripple. Note that all kernels except for $L 5$ have zero sums.

We have obtained the results by applying our proposed methods, Gabor, DCT, and Laws filters, to the synthetic textured images. The segmentation results are compared for five filter banks and are presented in the following section.

\subsection{Synthetic textured images}

To test the algorithm, a set of synthetic textured images formed by selected textures from the Brodatz album [20], MIT Vision and Modeling Database (see http://vismod.media.mit.edu/vismod), and MeasTex Image Texture Database (see http://www.cssip.uq.edu.au/meastex/ meastex.html) are used. We used combinations of 2, 3, 4, and 5 textures as test images. The sample synthetic images consist of D77, D84, D55, D53, D24, and D17 selected from the Brodatz album; Fabric.0000, Fabric.0017, Flowers.0002, Leaves.0006, and Leaves.0013 selected from MIT Vision and Modeling Database; and Grass.0002, Misc.0002, and Rock.0005 selected from MeasTex Image Texture Database.

The image presented in Figure $9 \mathrm{a}$ is a textured image consisting of Fabric.0000 from MIT Vision and Modeling Database. Figure $9 \mathrm{~b}$ is the true segmentation reference image. Figure $9 \mathrm{c}$ shows the segmented result by GCN. Figure $9 \mathrm{~d}$ is the segmentation result by AGFB. In Figures $9 \mathrm{e}$ and $9 \mathrm{f}$ the segmented result by DCT and Laws filters are depicted, respectively. The classification errors are presented in Table 2. As we can observe in this table, the classification errors are $0.59 \%$ and $2.57 \%$ for GCN and AGFB in comparison with $2.91 \%, 3.42 \%$, and $3.56 \%$ which are obtained by DCT, Laws, 


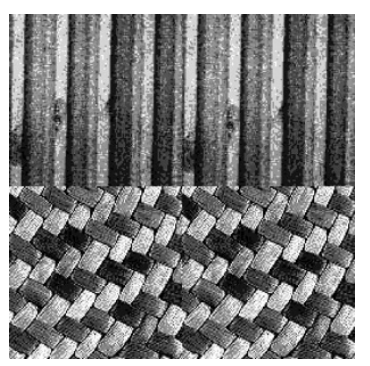

(a)

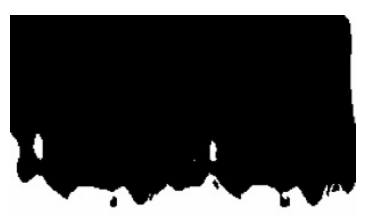

(d)
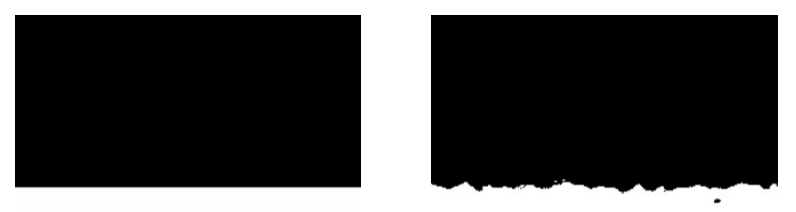

(b)

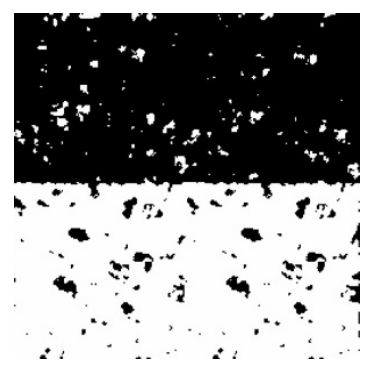

(e) (c)

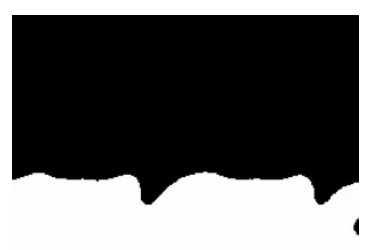

(f)

Figure 9: (a) Texture image. (b) The true segmentation reference image. (c) Segmentation results by GCN method; classification error is $0.59 \%$. (d) Segmentation results by AGFB; classification error is 2.57\%. (e) Segmentation results by DCT; classification error is $2.91 \%$. (f) Segmentation results by Laws filters; classification error is $3.42 \%$.

TABLe 2: Classification errors for Figure 8.

\begin{tabular}{cc}
\hline Method & Classification error \\
\hline GCN & $0.59 \%$ \\
AGFB & $2.57 \%$ \\
DCT & $2.91 \%$ \\
Laws & $3.42 \%$ \\
Gabor & $3.56 \%$ \\
\hline
\end{tabular}

and the Gabor filters, respectively. The GCN improves the classification results by $80 \%$ and $91 \%$ in comparison with the DCT and Laws filters, respectively. These amounts are 12\% and $58 \%$ for AGFB.

The image depicted in Figure 10a is a textured image consisting of Fabric.0000, Fabric.0017, Flowers.0002, Leaves.0006, and Leaves.0013 selected from MIT Vision and Modeling Database. Figure 10b is the true segmentation reference imag. Figure 10c shows the segmented result by GCN method and Figure 10d shows the segmented result by AGFB. The segmented results by Laws and DCT are depicted in Figures 10e and 10f, respectively.

The comparison of the classification errors using our proposed methods and the segmentation results using Gabor, DCT, and Laws filters for textured image of Figure 10 are shown in Table 3. The proposed GCN method improved the classification results by $45 \%$ and $79 \%$ in comparison with DCT and Laws filters, respectively. These amounts are 12\% and $58 \%$ for AGFB.

According to the results obtained in this study which are shown in Table 3, and the previous research, the proposed approaches have better classification performances in com- parison with the other three methods, that is, Gabor, DCT, and Laws filter bank. The filter parameters for different filter banks which are used for image segmentation are shown in Table 3. As depicted in Table 3, the number of filters for Gabor, DCT, and Laws are equal to the feature dimension and are 20,8 , and 25 , respectively. In GCN, despite having 42 filters in the filter bank, the feature dimension is reduced to 21 and in AGFB, the number of filters is adaptively selected.

\section{DISCUSSION AND CONCLUSION}

In this paper, two new methods for texture image segmentation are presented. The two proposed approaches optimize Gabor filter bank by achieving more accurate results, reducing the classification error, and reducing the filter bank size. In the first method (GCN), the classification error is reduced by implementing a Gabor filter bank with narrow angular bandwidth. Two low-frequency and high-frequency filters are added to improve the filter bank which made the total number of filters reach 42 . In this approach, neural networks is employed for both feature reduction and segmentation. A competitive network is combined with an MLP to learn the nonlinear relationship between quantized vectors and desired outputs. The second algorithm uses an energy ratio criterion to select the best discriminative filters adaptively (AGFB). A few Gabor filters are selected according to the energy ratio of each texture pair in an image. Extracted feature vectors which are obtained by applying the subset filter bank are classified using an MLP. Despite noticeable feature dimension reduction, this method provides better results than DCT and Laws filters. 


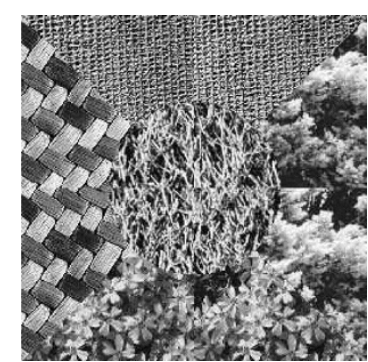

(a)

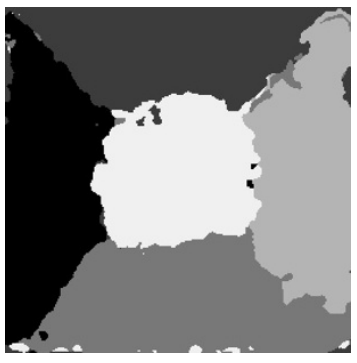

(c)

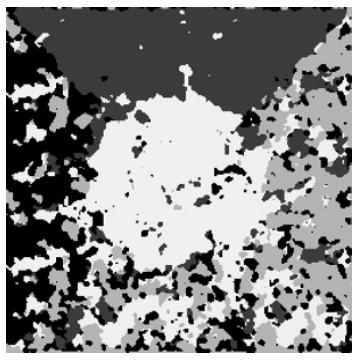

(e)

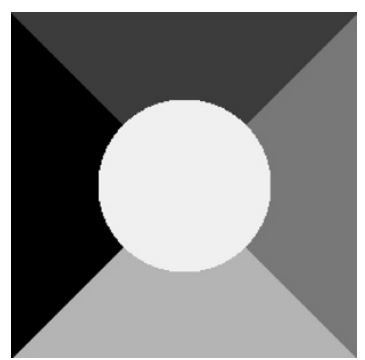

(b)

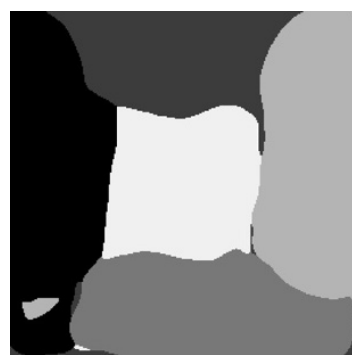

(d)

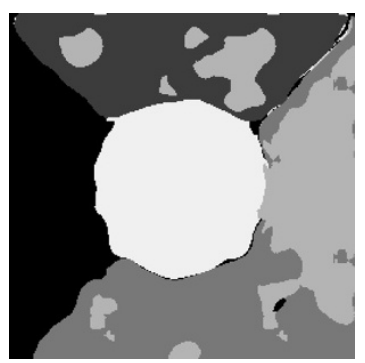

(f)

FIGURE 10: (a) Textured image. (b) The true segmentation reference image. (c) Segmentation results by GCN method; classification error is $8.37 \%$. (d) Segmentation results by AGFB; classification error is $12.62 \%$. (e) Segmentation results by Laws filters; classification error is $25.7 \%$. (f) Segmentation results by DCT; classification error is $15.27 \%$.

TABLE 3: Number of filters and features for Figure 9.

\begin{tabular}{cccc}
\hline Method & Segmentation improvement (percent) & No. of filters & No. of features \\
\hline GCN & 8.37 & 42 & 21 \\
AGFB & 12.62 & 10 & 10 \\
DCT & 15.27 & 8 & 8 \\
Laws & 25.7 & 25 & 25 \\
Gabor & 27.5 & 20 & 20 \\
\hline
\end{tabular}

The proposed segmentation algorithms are formulated as a combinational optimum approach to obtain a better filter bank, to reduce feature dimension, and to improve classification. The main advantages of the proposed methods include the following:

(1) the proposed approaches allow to use a larger filter bank consisting of a higher number of channels;

(2) both methods permit the use of a larger number of filter banks while the process compensates for the weakness of a specific filter bank in some frequencies;

(3) the feature quantization and classification component of this algorithm is a generic approach with the ability to learn and process different kinds of input vectors (feature vectors);

(4) both methods are capable of segmenting complex textured images.

Both GCN and AGFB produce better results for synthetic images than Gabor, DCT, and Laws filters. The Laws and DCT provide better results than Gabor filter bank with 20 filters [4], but our optimized Gabor filter banks using two different methods perform much better than DCT and Laws filter banks. On the other hand, the complexities of both 
proposed methods are reasonable and almost as complex as DCT and Laws filters. Among these five filter banks, AGFB is the fastest because of its small number of filters. As a tradeoff between the speed and classification error, both of our proposed methods offer more than a $50 \%$ improvement in classification. GCN takes almost the same computation time as 20 Gabor filters. Although the GCN method reduces the feature dimension using principal component analysis, implementing PCA by means of a competitive network speeds up the algorithm and is much faster than the conventional PCA.

Designing the Gabor filter bank by accurate parameter selection and using a different learning method according to the specific segmentation problem may improve the accuracy of this algorithm to get better segmentation results based on the nature of the input images. Minimizing the number of filters for texture discrimination has crucial importance and is still an area of active research. Thus, improving the AGFB method would be part of the future work. The algorithm may also be extended to problems such as biomedical and satellite image segmentation. Our current goal is to improve the performance of this algorithm and, in future research, apply the proposed techniques to segmentation of satellite and synthetic aperture radar (SAR) texture images.

\section{REFERENCES}

[1] A. C. Bovik, M. Clark, and W. S. Geisler, "Multichannel texture analysis using localized spatial filters," IEEE Trans. Pattern Anal. Machine Intell., vol. 12, no. 1, pp. 55-73, 1990.

[2] D. F. Dunn and W. E. Higgins, "Optimal Gabor filters for texture segmentation," IEEE Trans. Image Processing, vol. 4, no. 7, pp. 947-964, 1995.

[3] A. K. Jain and F. Farrokhnia, "Unsupervised texture segmentation using Gabor filters," Pattern Recognition, vol. 24, no. 12, pp. 1167-1186, 1991.

[4] T. Randen and J. H. Husoy, "Filtering for texture classification: a comparative study," IEEE Trans. Pattern Anal. Machine Intell., vol. 21, no. 4, pp. 291-310, 1999.

[5] I. Ng, T. Tan, and J. Kittler, "On local linear transform and Gabor filter representation of texture," in Proc. 11th IAPR International Conference on Pattern Recognition (ICPR '92), pp. 627-631, the Hague, the Netherlands, August-September 1992.

[6] K. I. Laws, "Rapid texture identification," in Conference on Image Processing for Missile Guidance, vol. 238 of Proceedings of SPIE, pp. 367-380, San Diego, Calif, USA, July 1980.

[7] D. A. Pollen and S. F. Ronner, "Visual cortical neurons as localized spatial frequency filters," IEEE Trans. Syst., Man, Cybern., vol. 13, no. 5, pp. 907-916, 1983.

[8] J. G. Daugman, "Uncertainty relation for resolution in space, spatial frequency, and orientation optimized by twodimensional visual cortical filters," Journal of the Optical Society of America $\{A\}$, vol. 2, no. 7, pp. 1160-1169, 1985.

[9] M. Unser and M. Eden, "Nonlinear operators for improving texture segmentation based on features extracted by spatial filtering," IEEE Trans. Syst., Man, Cybern., vol. 20, no. 4, pp. 804-815, 1990.

[10] T. P. Weldon, W. E. Higgins, and D. F. Dunn, "Gabor filter design for multiple texture segmentation," Optical Engineering, vol. 35, no. 10, pp. 2852-2863, 1996.

[11] D. A. Clausi and M. Ed Jernigan, "Designing Gabor filters for optimal texture separability," Pattern Recognition, vol. 33, no. 11, pp. 1835-1849, 2000.
[12] A. K. Jain and K. Karu, "Learning texture discrimination masks," IEEE Trans. Pattern Anal. Machine Intell., vol. 18, no. 2, pp. 195-205, 1996.

[13] T. P. Weldon and W. E. Higgins, "Design of multiple Gabor filters for texture segmentation," in Proc. IEEE Int. Conf. Acoustics, Speech, Signal Processing (ICASSP '96), vol. 4, pp. 22452248, Atlanta, Ga, USA, May 1996.

[14] A. C. Bovik, "Analysis of multichannel narrow-band filters for image texture segmentation," IEEE Trans. Signal Processing, vol. 39, no. 9, pp. 2025-2043, 1991.

[15] J. Strand and T. Taxt, "Local frequency features for texture classification," Pattern Recognition, vol. 27, no. 10, pp. 13971406, 1994.

[16] R. O. Duda and P. E. Hart, Pattern Classification and Scene Analysis, John Wiley \& Sons, New York, NY, USA, 1973.

[17] J. Beck, "Textural segmentation, second-order statistics, and textural elements," Biol. Cybernetics, vol. 48, no. 2, pp. 125$130,1983$.

[18] T. Kohonen, Self-Organizing Maps, Springer-Verlag, Berlin, Germany, 1997.

[19] J. A. Freeman and D. M. Skapura, Neural Networks: Algorithms, Applications and Programming Techniques, AddisonWesley, Reading, Mass, USA, 1992.

[20] P. Brodatz, Textures: a Photographic Album for Artists and Designers, Dover Publications, New York, NY, USA, 1966.

Nezamoddin N. Kachouie received his B.S. degree in computer engineering from Isfahan University of Technology (IUT) in 1989 and his M.S. degree in electrical and computer engineering from Ryerson University in 2003. Currently he is a Ph.D. candidate at the University of Waterloo. His research area is biomedical video segmentation and tracking. Currently he is modeling and designing an automatic-model-based segmentation and tracking system with the applications of multicellular microscope cell images. He is interested in deformable models and Bayesian framework for biomedical applications of multiple-target tracking. His research interests include object tracking, spatiotemporal image analysis, image denoising, optimization, and classification, and applications of fuzzy logic, neural networks, and genetic algorithms in image processing.

Javad Alirezaie received his B.S. degree in electrical and electronic engineering from Tehran University in 1988 and his M.A.Sc. and Ph.D. degrees in systems design engineering from the University of Waterloo in 1993 and 1996, respectiv+ely. From 1997 to 2000, he was a Postdoctoral Fellow, an Assistant Professor, and an R\&D Fellow in a private sector. He joined Ryerson University in 2001. He is currently an Assistant Pro-

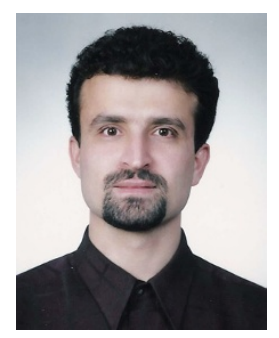
fessor in the Department of Electrical Engineering and an Adjunct Professor with the Department of Systems Design Engineering, University of Waterloo. His research interests include medical imaging, signal and image processing, neural networks, speech processing, and pattern recognition. He is the author and coauthor of several technical papers in these areas. Dr. Alirezaie is a licensed Professional Engineer (P.Eng.), and a Member of IEEE, IASTED, and SPIE Societies. 